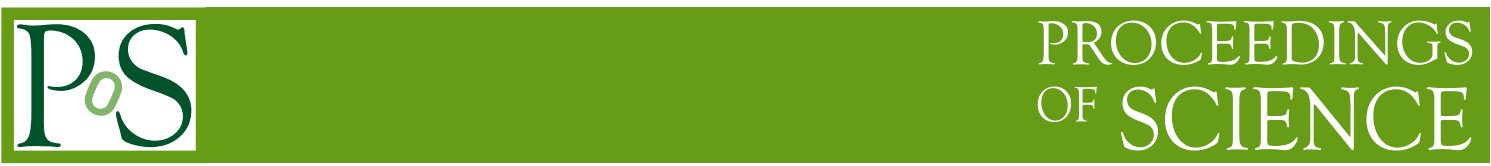

\title{
Short Gamma Ray Bursts detected by INTEGRAL
}

\author{
S. Meehan*, M. Topinka, A. Martin-Carrillo, R. O’Connor, L. Hanlon, S. Foley and \\ B. McBreen \\ UCD School of Physics, University College Dublin, Ireland \\ E-mail: seamus.meehan@ucdconnect.ie, martin.topinka@ucd.ie, \\ antonio.martin-carrillo@ucd.ie, lorraine.hanlon@ucd.ie, \\ sfoley@bermuda.ucd.ie, brian.mcbreen@ucd.ie
}

The INTEGRAL Burst Alert System (IBAS) has detected and localised 71 Gamma-Ray Bursts (GRBs) as of January 2010. Of these only three have a $\mathrm{T}_{90}$ duration less than 2 seconds. Short GRBs are believed to be produced in the merger of compact objects such as neutron stars and black holes. The short bursts detected by INTEGRAL are GRB 070707, GRB 071017 and GRB $081226 \mathrm{~B}$ with values of $\mathrm{T}_{90}$ of $0.8,0.4$ and 0.4 seconds respectively. There are no redshift determinations for any of these bursts. GRB 070707 and GRB 081226B are spectrally hard bursts which is consistent with the short GRB classification. GRB 071017 has a much softer spectrum and is located within 0.25 degrees of the galactic plane indicating that it may be a galactic transient such as Swift J195509+261406 associated with GRB 070610. We estimate the rate of these galactic transients as $\sim 2$ per year. The temporal and spectral properties of the three bursts are presented including peak flux, fluence and photon indices.

The Extreme sky: Sampling the Universe above $10 \mathrm{keV}$ - extremesky2009,

October 13-17, 2009

Otranto (Lecce) Italy

\footnotetext{
* Speaker.
} 


\section{Introduction}

Gamma-Ray Bursts (GRBs) are extremely powerful transient sources observed a few times per day throughout the whole sky. Their short durations and highly variable temporal structures provide constraints on the central engines of these bursts. GRBs are classified into two different groups determined by their value of $\mathrm{T}_{90}$. The $\mathrm{T}_{90}$ of a burst is defined as the time during which the middle $90 \%$ of the gamma ray counts are detected. This classification of GRBs is based on the observed bimodal distribution of number of bursts versus $\mathrm{T}_{90}$ [1]. GRBs with $\mathrm{T}_{90}$ above 2 seconds are classified as long while those with $\mathrm{T}_{90}$ below two seconds are classified as short. Short bursts on average have higher hardness ratios than long GRBs. Two main theories have been proposed to explain the processes involved in the formation of long and short GRBs. Long bursts are believed to be produced in the collapse of massive stars in type Ic supernovae while short bursts are thought to be the result from the merger of compact objects [2;3]. The merger of two compact objects will produce a rapidly spinning black hole with a compact accretion disk. The binding energy of the accretion disk along with the rotational energy of the black hole are the main energy reservoirs. The energy is then released quickly according to the fireball model and collimated into a pair of jets. The shocks within these jets produce the observed gamma rays. A comparison between short and long BATSE bursts showed that most short bursts are spectrally harder than long bursts [4]. The temporal properties of both the short and long GRBs are consistent with lognormal distrubutions $[5 ; 6]$.

\section{Observations with INTEGRAL}

There are two main gamma ray instruments on board INTEGRAL, IBIS and SPI, which are optimised for high resolution imaging and spectroscopy of the gamma ray sky. IBIS [7] is a coded mask instrument comprised of two detectors planes, ISGRI [8] and PICsIT [9], in the energy range $14 \mathrm{keV}-1 \mathrm{MeV}$ and $180 \mathrm{keV}-10 \mathrm{MeV}$ respectively. IBIS/ISGRI has $16384 \mathrm{CdTe}$ detectors, located $3.4 \mathrm{~m}$ from a tungsten coded mask. Images of the sky are reconstructed by decoding the shadow cast using the mask pattern. The field of view of IBIS is $9^{\circ} \times 9^{\circ}$ fully coded, $19^{\circ} \times 19^{\circ}$ at $50 \%$ coding and $29^{\circ} \times 29^{\circ}$ at zero coding. SPI [10] is also a coded mask instrument which consists of 19 cooled Germanium detectors and covers the energy range from $20 \mathrm{keV}-8 \mathrm{MeV}$. It has a high energy resolution of $2.5 \mathrm{keV}$ at $1.3 \mathrm{MeV}$. The coded mask on SPI is located $1.71 \mathrm{~m}$ above the detector plane and the corner-to-corner fully coded field of view is $16^{\circ}$. This increases to $34^{\circ}$ at 50\% coding. The Integral Burst Alert System (IBAS) [11] is an automatic ground based system for accurate localisation of GRBs and rapid distribution of the coordinates. The analysis was carried out using the Offline Scientific Analysis (OSA) version 5.0.

\section{Results}

As of January 2010, INTEGRAL has detected and localised 71 GRBs [12; 13]. Of these, only three fit the criteria for short GRBs. The next shortest bursts GRB 040422 and GRB 050922 have $\mathrm{T}_{90}$ values of 4 and 8 seconds respectively. However they lack the usual spectral hardness associated with short GRBs. The light curves and spectra of the three GRBs are given in Figure 1 and the properties of the bursts are summarised in Table 1. 
(a)

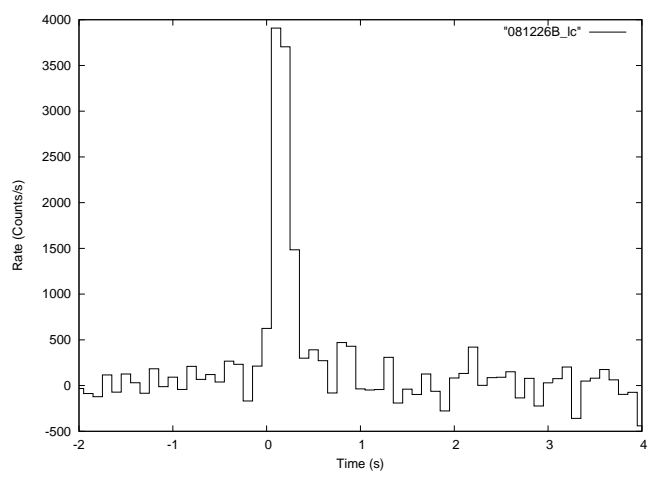

(c)

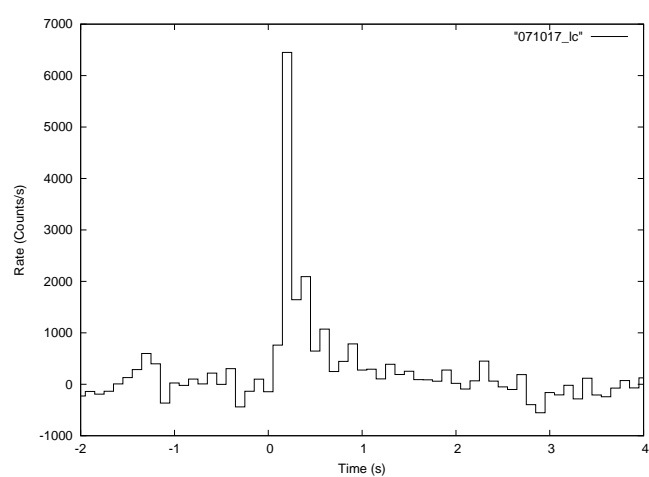

(e)

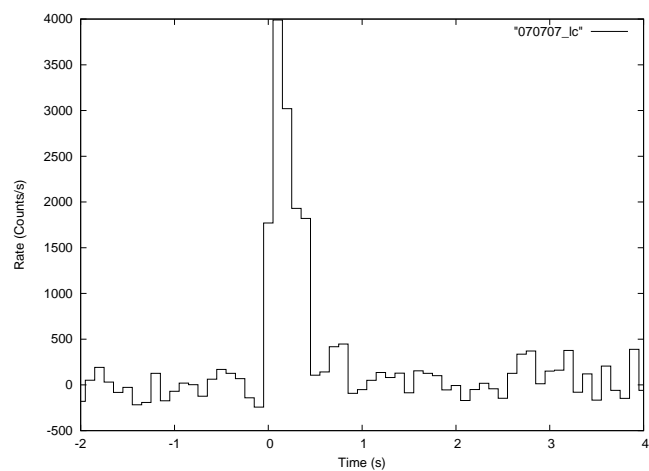

(b)

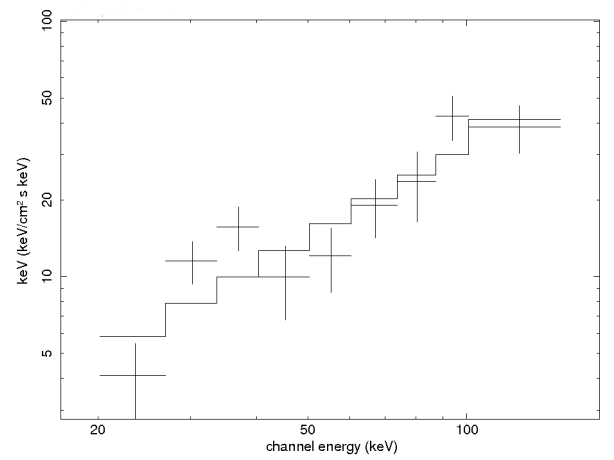

(d)

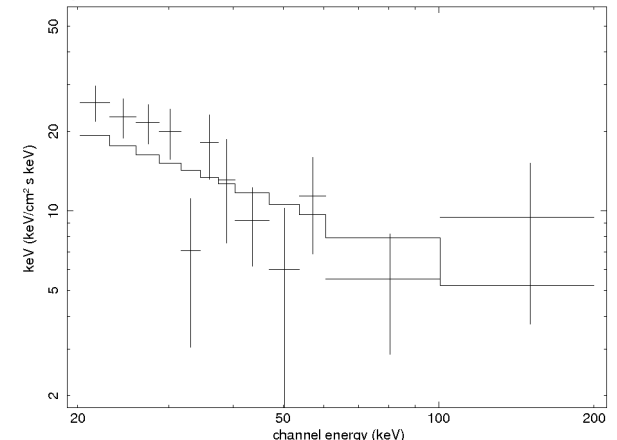

(f)

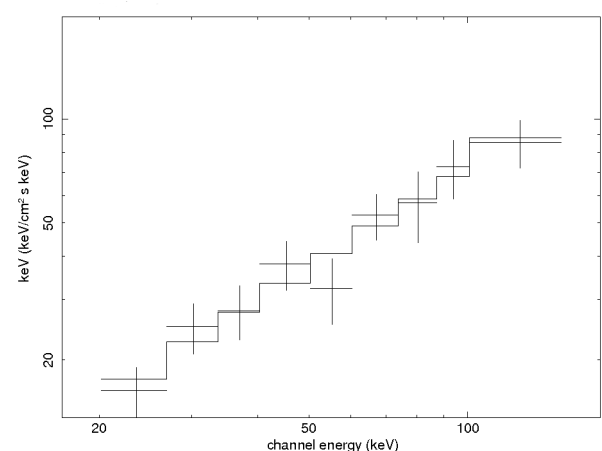

Figure 1: Figures (a), (c) and (e) show the lightcurves over the 20-200 keV range for GRBs 081226B, 071017 and 070707 respectively. Figures (b), (d) and (f) show the $v \mathrm{~F}_{v}$ spectra fit with power laws for GRBs $081226 \mathrm{~B}, 071017$ and 070707 respectively also in the $20-200 \mathrm{keV}$ energy range. There is a large difference between the spectrum of GRB 071017 and the other two bursts.

\subsection{GRB 081226B and GRB 070707}

GRB 081226B is a spectrally hard burst as shown by the photon index (Table 1). It is therefore consistent with typical short hard GRBs. The burst was also detected by the Gamma-ray Burst Monitor (GBM) onboard Fermi [14]. It was 22 degrees from the LAT boresight but no detection has been reported. The INTEGRAL error region was also observed by the Swift X-ray Telescope (XRT) $9.8 \mathrm{ks}$ after the burst but no afterglow was detected [15]. No host galaxy has been identified 


\begin{tabular}{|l|r|r|r|}
\hline & GRB 081226B & GRB 071017 & GRB 070707 \\
\hline $\mathrm{T}_{90}$ (seconds) & 0.4 & 0.4 & 0.8 \\
\hline Photon Index & $-0.98_{-0.64}^{+0.69}$ & $-3.00_{-0.61}^{+0.77}$ & $-1.19_{-0.13}^{+0.14}$ \\
\hline Peak Flux & $1.38_{-0.40}^{+0.16}$ photons cm $\mathrm{s}^{-1}$ & $1.39_{-0.51}^{+0.02}$ photons cm $^{-2} \mathrm{~s}^{-1}$ & $1.79_{-0.21}^{+0.06} \mathrm{photons} \mathrm{cm}^{-2} \mathrm{~s}^{-1}$ \\
\hline Fluence & $\left(1.86_{-0.62}^{+0.04}\right) \times 10^{-7} \mathrm{ergs} \mathrm{cm}^{-2}$ & $\left(6.68_{-1.45}^{+0.08}\right) \times 10^{-8} \mathrm{ergs} \mathrm{cm}^{-2}$ & $\left(2.07_{-0.32}^{+0.06}\right) \times 10^{-8} \mathrm{ergs} \mathrm{cm}^{-2}$ \\
\hline Coordinates & & & \\
RA, DEC & $1: 41: 58.8,-47: 26: 20$ & $8: 18: 52,-16: 00: 03$ & $17: 51: 00,-68: 52: 52$ \\
Galactic & $280.28,-67.40$ & $15.02,-0.25$ & $324.65,-19.98$ \\
\hline
\end{tabular}

Table 1: Properties of the three short Gamma Ray Bursts detected by INTEGRAL

for this burst. GRB 070707 was the first short GRB detected by INTEGRAL (McGlynn et al 2008). Swift did not trigger on GRB 070707 because the burst occurred outside the BAT field of view and no observations were made until 8.8 hours after the burst. The XRT observed the GRB location and detected a source at R.A $=17 \mathrm{~h} 50 \mathrm{~m} 58.49 \mathrm{~s}$, Dec $=-68^{\circ} 55^{\prime} 27^{\prime \prime}$ with an uncertainty of $5.4^{\prime \prime}$ at the $90 \%$ confidence level, within the INTEGRAL error region. The XRT carried out a further observation between 325 and $517 \mathrm{ks}$ after the trigger, but the source had faded to below the XRT detection limit [17]. It was not possible to estimate a break time from the X-ray afterglow lightcurve. Optical observations carried out using the VLT identified a host galaxy for the GRB with a magitude of 27.3 and an upper limit on the redshift of 3.6 [18].

\subsection{Comparison between GRB 071017 and GRB 070610}

GRB 070610 was detected by the Swift Burst Alert Telescope (BAT). An uncatalogued source was found by the Swift X-ray Telescope (XRT) at a position of RA=19h 55m 09.6s, Dec $=+26^{\circ}$ $14^{\prime} 06.3^{\prime \prime} 3.2 \mathrm{ks}$ after the BAT trigger [19]. These coordinates are approximately 1 degree from the galactic plane. The detected source was designated Swift J195509+261406 and observations showed flaring in the X-Ray, optical and infra-red bands [20; 21]. Optical flares were detected up until 3 days after the burst when the activity decayed abruptly. No further flares were detected until 12 days after the burst when a late time lower brightness near infra-red flare was detected (Stefanescu et al, 2008). While several candidates were considered for the progenitor of this source, the most likely candidate is a new galactic magnetar [20]. The power law index of the time-averaged spectrum of GRB 070610 was measured to be $-1.76_{-}^{+} 0.25$.

GRB 071017 has a photon index of approximately -3 which is considerably softer than expected for a short burst (Figure 1) and also softer than GRB 070610. It was detected 0.25 degrees from the galactic plane (Table 1). The Swift XRT observed the error region of GRB 0710172.3 days after the burst for a duration of $2 \mathrm{ks}$. A source was detected within the error region which was consistent with the XMM source 2XMM J171751.2-155920 [22] and no flaring was detected in this source. Unfortunately there are very few reported follow up observations of this burst and hence a flaring phase of the type observed in Swift J195509+261406 cannot be excluded. 


\section{Discussion}

The isotropic peak luminosity can be calculated using the 50-300 keV peak flux. The peak flux in this band is $2 \times 10^{-7} \mathrm{ergs} \mathrm{s}^{-1}$ for GRB 070707 . Assuming an average redshift for short GRBs of $\mathrm{z}=0.35$ [2], we obtain a peak luminosity of $\mathrm{L}_{\text {peak }}=1.1 \times 10^{50} \mathrm{ergs} \mathrm{s}^{-1}$.

The spectral properties of the prompt emission can be used to estimate a lower limit on the bulk Lorentz factor $\Gamma$ of the $\gamma$-ray source [23]. The prompt emission of short GRBs is predominantly non-thermal, including the spectrum of GRB 070707. This implies that the $\gamma$-ray source is optically thin to Thomson scattering on electron-positron pairs [24]. If the optical depth is $\tau_{T}<1$ regardless of whether internal or external shocks are involved in generating the emission, a lower limit on $\Gamma$ can be estimated using the fit to the $\gamma$-ray spectrum in the equation:

$$
\left(\frac{\Gamma m_{e} c^{2}}{E_{\text {peak }}(1+z)}\right)+(4-\alpha) \ln \Gamma+\ln \left(\frac{E_{\text {peak }}}{m_{e} c^{2}}\right) \geq 30
$$

where $\alpha$ is the photon index and $E_{\text {peak }}$ is the peak energy of a cutoff power law fit. The cutoff power law gave a value of $E_{\text {peak }}$ of $645_{-330}^{+766} \mathrm{keV}$ Assuming a redshift of z $=0.35$ we get a lower limit in the range of $\Gamma \geq 15-40$. We can see from this $\Gamma$ that short GRBs are ultra-relativistic. A similar value of $\Gamma$ is obtained for GRB 081226B.

This is comparable to the lower limits calculated by Nakar [23] for two other short GRBs, $\Gamma \geq$ 4 for GRB 050709 and $\Gamma \geq 25$ for GRB 051221a. The relatively low Lorentz factor implies a late deceleration time and a smaller initial radius, resulting in the possible late onset of the afterglow.

\section{Conclusions}

The spectral and temporal properties of GRB 070707 and GRB 081226B are in agreement with the properties of short bursts detected by other gamma-ray satellites. While GRB 071017 has similarities to Swift J195509+261406 there is no conclusive evidence that these sources are related because of the lack of multi-wavelength observations of GRB 071017. We estimate a rate of $\sim 2$ galactic transients per year given the time spent by Swift observing the galactic plane. This estimate is consistent with the probable detection of one galactic transient by INTEGRAL to date.

\section{References}

[1] Kouveliotou et al, 1993, ApJ, 413, L101

[2] Nakar, E., 2007, Phys.Rept, 442, 166-236

[3] Gehrels, N., Ramirez-Ruiz, E. and Fox, D., 2009, Annual Review of Astronomy and Astrophysics, 47, 567-617

[4] Ghirlanda et al, 2004, A\&A, 422, 155

[5] McBreen, S. et al, 2001 A\&A 380, L31

[6] Quilligan, F et al, 2002 A\&A 385, 377 
[7] Ubertini, P., Lebrun, F., Di Cocco, G. et al, 2003, A\&A, 411, L131

[8] Labanti, C., Di Cocco, G., Ferro, G., et al. 2003, A\&A, 411, L149

[9] Lebrun, F., Leray, J. P., Lavocat, P., et al. 2003, A\&A, 411, L141

[10] Vedrenne, G., Roques, J.-P., Schonfelder, V., et al. 2003, A\&A, 411, L63

[11] Mereghetti, S. et al, 2003, A\&A, 411, L291

[12] Foley, S. et al, 2008, A\&A 484, 143-157

[13] Vianello, G., Gotz, D., Mereghetti, S. A\&A 2009

[14] E. Bissaldi et al, 2008 GCN 8751

[15] Evans, P. \& Hoversten, E., 2008, GCN 8740

[16] McGlynn, S. et al, 2008 A\&A, 486, 405M

[17] Beardmore A. \& Parsons, 2007, GCN 6610

[18] Piranomonte, S. et al, 2008, A\&A 491, 183-18

[19] Pagani, C. et al, 2007 GCN 6490

[20] Castro-Tirado, A. J. et al, 2008, Nature 455, Issue 7212, pp. 506-509

[21] Stefanescu, A. et al, 2008, Nature 455, 503-505

[22] Evans, P.A. et al, 2007, GCN 6942

[23] Nakar, E., 2007b, Adv. SpaceRes.40:1224-1228

[24] Lithwick, Y. \& Sari, R., 2001, ApJ, 555, 540 